\title{
Where has fundamental physics gone?
}

Can an excess of success in solving problems have induced physicists to overlook the serious intellectual difficulties that surround them?

IN Aristotle's time, nearly 3,000 years ago, there was still everything in science to play for. To have the wit to ask what sustains the motion of a projectile, say a thrown stone, was a triumph in itself. Aristotle's explanation that a projectile will create a void into which air will rush, impelling the projectile on its course, has the merit of seeming consistency, but no other. Yet, by drawing attention to conceptual issues, it did much to keep succeeding millennia on their toes.

So, for that matter, did Einstein, with $E=m c^{2}$. Since then, of course, there have been general relativity, quantum mechanics, nuclear structure, the recognition of Hubble motion in an expanding Universe, quantumelectrodynamics more generally, and even ambitions that all four forces of nature (if that is how many there are) will be unified. So why has physics as practised (or, at least, as published) boiled down to mere problem-solving?

Aficionados will deny the slur, but they will be wrong. Those who even occasionally look at this space will have been given the impression that there is no better guide to what is interesting and important in physics than Physical Review Letters, the weekly journal of the American Physical Society. The impression is correct. But the grand themes of Aristotle and Einstein seem to have given ways to declarations such as "arrays of coupled limit-cycle oscillators are of fundamental importance in physics, biology and engineering" and "The existence of isospectral $\gamma$ sequences in a number of neighbouring superdeformed nuclei poses an exciting challenge to nuclear theorists". Each statement is the first sentence of an article in Physical Review Letters for 11 November, chosen at random. Where are the grand themes of the old days? And why have they disappeared? To be fair, nobody in his or her right mind would expect that every paper in a journal running to more than 140 pages a week would raise a fundamentally novel question. But should not some of them do that?

Pedestrianism, sadly, seems to have taken over. So much can be gathered from the way in which people sidle up to the question of whether there can be a correspondence between the stable states (eigenstates) of quantum systems and the states in which the corresponding classical systems are prone to what is called 'deterministic chaos'.

Evidently it would be a question of
Aristotelian grandeur to ask whether the correspondence is invariable and in which directions the words 'necessary' and 'sufficient' apply. But the practitioners seem more eager to solve problems, sometimes reporting how much CPU time they have used in their numerical solutions. We (the readers) are still awaiting news of whether classical chaos means an eigenstate, or an eigenstate means classical chaos, necessarily or sufficiently as the case may be.

The disappointments seem widely spread. In the previous issue of the same journal, more than 200 people describe an experiment at Fermilab that failed to detect the decay of charged heavy vector bosons (elaborations of the intermediate vector bosons $\mathrm{W}^{ \pm}$and $\mathrm{Z}^{0}$ ) into leptons, leading them to conclude that such particles will not be found with any accelerator now being built. And a small army of Japanese showed that the decay of the strange $\mathrm{K}^{0}$ meson into positive and negative pions is so much more common than their decay into muons and electrons that these processes can be neglected as literally insignificant. Nobody will be surprised; it would have been different if the experiments had turned out differently.

Understandably, in the circumstances, there seems a great deal of fine tuning under way. In an earlier issue, there is a scholarly but inconclusive discussion of whether there are neutrinos with a mass of $17 \mathrm{keV}$ and some experiments that show that highly excited caesium and rubidium atoms (in Rydberg states) behave as if they were chaotic systems.

Little things irritate, and are bound to do so, when there is so little grand to say. But it is more than a passing inconvenience that readers should be told as often as they are that a crucial reference has, as yet, no citation, but only the phrase "to be published". It is especially mean that this should apply to pieces of algebra.

Inevitably, what catches the eye (and sticks in the mind) during times of ennui such as this is experimental neatness. Luckily (to judge from Physical Review Letters) a lot of that persists.

Take, for example, the case of the group at the University of Colorado at Boulder that has devised a novel way of storing particles (such as atoms) that have a magnetic moment. Using the absorption lines of, say, caesium, atoms are first 'cooled' by means of six off-time laser beams (one from each face of a surrounding cube) in what is now a relatively familiar trap of 'optical molasses'. When the optical trap is filled, the atoms are then propelled vertically upwards, through exactly 14 centimetres, into what is nothing but an axially symmetric bottle oscillating between axial and radial confinement so that the atoms are further 'cooled', or robbed of kinetic energy. The trap is an obvious means of dealing with spin-polarized atoms. The group promises next to measure something.

There are also several neat ideas put into circulation. What happens, for example, to an atom adsorbed on a crystalline surface of identical composition?STM measurements have now made it clear that even apparently perfectly flat surfaces are marked by ledges, just one atomic diameter high, where single layers of atoms come to an edge. An atom adsorbed below a ledge will, by diffusion, eventually unite with it, but one adsorbed above a ledge is much less likely to tumble over, and so will be a roughening influence.

The same issue of the same journal has an account of a humble study of the aggregation of polar molecules on a water surface. Circular patches become hexagonal when the aggregation rate is right. The explanation has to do with the lesser surface free energy of a shape whose curvature is concentrated at the corners. Is that why snowflakes are hexagonal?

Interesting though it may be, all this is the small change of physics, hardly worthy of the attention of an Aristotle or of an Einstein. Physics is going through a dull patch, when even predicted attainments, such as the discovery of the top quark, will merely bring relief (if they are made) and a clamour for bigger accelerators (if they are not).

Although it would be irresponsible to suggest that physics should manufacture ideological controversy to keep itself in the public eye, the more serious danger is that its élan may be destroyed by the complacency that problem-solving brings. And that is an odd conclusion from too much reading of an excellent journal.

John Maddox

\section{Correction}

LAST week's News \& Views leading article (Nature 354, 183; 1991) attributed to Joseph Goldstein the astounding allegation that half of all cytoplasmic proteins may be prenylated: the figure he actually named was 0.5 per cent. It should also have referred to lipid prenyl tails, not lipid tails. Our apologies to Dr Goldstein and to our readers. 